\title{
The undisclosed role of anoxia/hypoxia and disturbed sleep on glucose metabolism
}

\author{
Patrizio Tatti ${ }^{1^{*}}$, Desiderio Passali ${ }^{2}$, Luisa Passali ${ }^{2}$ \\ ${ }^{1}$ Diabetes and Endocrinology Unit, Azienda Sanitaria Locale Roma “H”, Roma, Italy; *Corresponding Author: info@patriziotatti.it \\ ${ }^{2}$ ENT Department, University of Siena, Siena, Italy
}

Received 14 January 2012; revised 17 February 2012; accepted 19 March 2012

\begin{abstract}
Up to recently the respiratory disturbances were considered a risk factor for hypertension, but there was no trace of a role in metabolic disorders. Only recently the existence of a connection among the respiratory disturbance caused by the obstruction of the upper airways and a wide array of metabolic disturbances has been demonstrated. The respective roles of anoxial hypoxia and the attendant disturbed sleep remain, however, to be clarified.
\end{abstract}

Keywords: Diabetes Mellitus; OSAS; Sleep

Disturbance

\section{INTRODUCTION}

Up to recently the respiratory disturbances were considered a risk factor for hypertension, but there was no suggestion of a role in metabolic disorders. The existence of a connection between respiration and function of the metabolic machinery has been discovered in the last few decades. Both the patency of the upper respiratory airways and the metabolic functions are critical to survival and thus is no surprise that they may go hand in hand. The nasopharyngeal obstruction can occur at different anatomical levels and can be caused by many pathological processes. The most common are allergic and nonallergic rhinitis, turbinates hypertrophy, severe septal deviation, nasal polyposis, adenoid/tonsillar hypertrophy and oral anatomic alterations. The obvious and dramatic consequences are heavy snoring and the dangerous Obstructive Sleep Apnea Syndrome (OSAS). Fortunately topical, surgical and pharmacological interventions on nasal pathology are available to reverse symptoms and signs of both snoring and OSAS [1-4].

The metabolic functions in the body are strictly regulated. The most critical and well studied function is the regulation of the blood level of glucose.

The blood glucose serves an essential function in the body supplying the fuel for the cells. The relevance of this fuel is such that the body has a redundancy of mechanisms to avoid the dramatic and even lethal hypoglycemia: 1) Exogenous supply through food; 2) A liver reservoir that can be mobilized when needed through the process of glycogenolysis; 3 ) Turning aminoacids and fat into glucose (neoglucogenesis).

We currently know eight regulators of the blood glucose level and the delivery system to the cells elegantly defined the "ominous octet" by Ralph DeFronzo [5] (Table 1). Like any complex regulatory system, there are possible background "noises" interfering with the main signals. Recent research reveals that disturbed respiration and poor sleep could be potent interferences [6,7]. While the relationships of sleep with blood pressure, central nervous system, the respiratory system and hormonal secretion are widely recognized, the role of disturbed respiration/disturbed sleep on blood glucose control remains ignored. One of the main problems is that disturbed sleep and sleep disordered breathing (SDB)/ OSAS are so intimately connected that single out the role of each is an extremely difficult task. In this paper we review the main evidence connecting poor sleep with metabolic disorders.

\section{MAIN STUDIES}

We searched the available literature for papers with

Table 1. The ominous octet [1].

\begin{tabular}{cc}
\hline Organ involved & Effect on glucose metabolism \\
\hline Pancreas $\beta$ cell & Insulin secretion \\
Pancreas $\alpha$ cell & Glucagon secretion \\
Liver & neoglucogenesis \\
Kidney & Increased glucose reabsorption \\
Adipose tissue & Accelerated lipolysis \\
Gut & Incretin (GLP1) deficiency/resistance \\
Muscle & Glucose metabolism \\
Brain & Insulin resistance \\
\hline
\end{tabular}


clinical and epidemiological relevance. Spiegel et al. in 1999 [8] assessed carbohydrate metabolism, thyro-tropic function, activity of the hypothalamo-pituitary-adrenal axis, and sympathovagal balance in 11 young men after time in bed had been restricted to $4 \mathrm{~h}$ per night for 6 nights. These authors compared the sleep-debt condition with measurements taken at the end of a sleep-recovery period when participants were allowed $12 \mathrm{~h}$ in bed per night for 6 nights, and found that Glucose tolerance was lower in the sleep-debt condition than in the fully rested condition $(p<0.02)$, as were thyrotropin concentrations $(p<0.01)$. Under the stressful conditions evening cortisol concentrations ( $p=0.0001$ ), and activity of the sympathetic nervous system increased $(p<0.02)$. This was one of the first studies to demonstrate a role of sleep debit on glucose metabolism. Furthermore these Authors demonstrated that during sleep deprivation, there is 30\% reduction of the first peak of insulin response to a glucose load, that reverts to normal after restoration of the normal sleeping pattern. This observation is extremely interesting because the absence of this first peak of insulin secretion is a characteristic of the early diabetes mellitus [9].

There are not many studies on the subject of disturbed sleep/sleep apnea and blood glucose control and most of them lack sophistication or include a small number of patients, the observations are limited to one or few night's sleep and the role of the oxygen deprivation versus the number of overnight awakenings is not clear. A recent meta-analysis on 107,756 subjects participating in 10 studies reported an association between sleep quality assessed with a questionnaire and the risk of diabetes [10]. To the contrary experience on 70,026 women enrolled in the Nurses Health Study, indicated that the relationship between self reported sleep duration and incident diabetes was probably due to the confounding effect of Body Mass Index or weight gain [11]. In a recent paper Tasali et al. induced all-night selective suppression of slow wave sleep (SWS), without change in total sleep time. This intervention resulted in a marked decrease in insulin sensitivity without adequate compensatory increase in insulin release, leading to reduced glucose tolerance and augmented diabetes risk [12]. More recently the sleep Research center of Pennsylvania evaluated 1741 subjects of both sexes, using both the results from the sleep laboratory and questionnaires. This study demonstrated that insomnia with short sleep duration was associated with increased odds for diabetes [13]. Although this study has merits, the presence of sleep disorders was based on a standard questionnaire completed by the subjects which has an inherent uncertainty. Moreover if the questionnaires were self administered or completed with the help of the medical staff is not stated, but this aspect may have a considerable bearing on the results. In a more sophisticated study with indirect calorimetry and a double radar detection of physical activity, 31 obese diabetics were more sedentary during the day and had 59\% higher spontaneous physical activity during sleeping than 61 nondiabetic obese control subjects. The Authors explain this with the restlessness from snoring [14].

Doubtless impaired respiration/OSAS are strictly related to sleep performance, but the available studies fail to give definitive proof of the involvement of oxygen deficit on blood glucose control. There are however some papers pointing to a role of OSAS. Tiihonen demonstrated that insulin resistance is related to the severity of sleep apnea on 8 patients who underwent an overnight polisomnographic recording and an OGTT [15]. That OSAS can induce insulin resistance was demonstrated in a recent study of 118 nondiabetic males who underwent polysomnography and the Frequent Sampled Intravenous GlucoseTolerance test (FSIGIT), a test that can reveal subtle abormalities of insulin sensitivity [16]. In these subjects, compared with those without OSAS the insulin sensitivity was reduced respectively of $27 \%, 37 \%, 48 \%$ according to the presence of slight, moderate, or severe OSAS [17]. In another study of 150 men the Authors demonstrated that an increase in Apnea/hypopnea index was associated with increased risk of Impaired Glucose Tolerance and increased insulin resistance. This impairment was apparently related to the severity of Oxygen desaturation [18]. A 12-year follow-up of 2663 middle aged Swedish subjects demonstrated a high incidence of diabetes in men with sleep complaints or short sleep duration. Unfortunately this study was entirely based on postal questionnaires obtained at 12 years distance. While this study is relevant its design presents obvious limitations [19]. Another recent cross sectional analysis of $>2500$ OSAS non diabetic subjects demonstrated a significantly high prevalence of prediabetes and incident diabetes independent of obesity [20]. A group from Chicago recently presented the results of a study of 60 consecutive diabetic outpatients who underwent a polysomnography and HbA1c assay. In these subjects increasing severity of OSAS was associated with poorer glucose control, independent of adiposity and other confounders, with effect size of $1.49 \%$ [21]. An interesting study with pulse oxymetry of 4398 Japanese OSAS subjects followed for average 3 years for the development of diabetes, demonstrated a non statistically significant increased risk ratio of 1.26 in those with mild-intermittent hypoxia and a significant 1.69 for those with moderate-severe hypoxia [22]. Another indirect and intriguing observation comes from the Accord study. This study was started to confirm a positive effect of the intensive glucose lowering therapy on the cardiovascular outcome of diabetics, and was interrupted due to an unexpected excess of deaths in the intensive group. 
Interestingly this group showed an increase in weight, that may have aggravated the respiratory difficulties and with this mechanism increased the mortality [23]. There is evidence that the presence of OSAS causes an increase in the inflammatory cytokines, mostly TNF-alpha, that in turn can cause further increase in insulin resistance and atherosclerotic cardiovascular damage [24-26]. In a case control study, thirty OSA subjects were found to have a significantly more adverse vascular risk factor profile than 30 matched non-OSA subjects [27]. This was confirmed in another more recent matched case-control study of 42 OSAS subjects with increased insulin resistance and other risk factors for vascular disease [28]. Interestingly the inflammatory cytokines appear to have a role in causing pancreatic B-cell damage $[29,30]$. In summary, these studies can give sufficient proof of a connection between sleep disturbances, respiratory distress and glucose metabolism. Sleeping disordered breathing/ OSAS is also connected with obesity, which in turn is a factor in co-causing or worsening diabetes (Figure 1). Out of 773 OSAS subjects studied by Harsh and coworkers only 48 had a normal body weight, 174 were overweight and the remaining 551 were frankly obese [31]. Among these the OSAS subjects were particularly prone to gain weight [32]. Apparently sleep can modulate appetite through the hormonal environment and the hormone leptin [26]. This hormone is one of the main regulators of body weight and has a primary role in appetite control [33]. A recent randomized, 2-period, crossover clinical study of 12 healthy men explored daytime profiles of plasma leptin and ghrelin levels and subjective ratings of hunger and appetite. The observation was protracted for 2 days of sleep restriction and 2 days of sleep extension under controlled conditions of caloric intake and physical activity. The analysis of the data demonstrated that short sleep duration in young, healthy men is associated with decreased leptin levels, increased ghrelin levels, and increased hunger and appetite [34]. Another multicentric longitudinal cohort

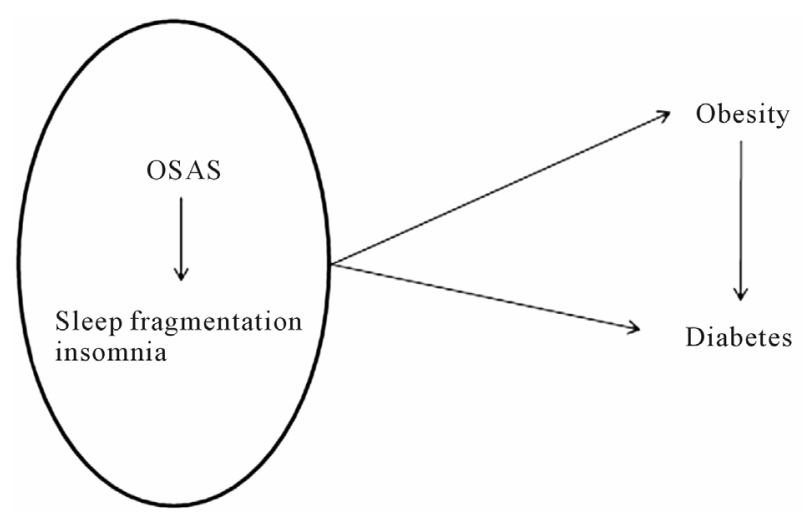

Figure 1. A simplified scheme of the interactions between respiratory distress and metabolic disorders. study of the cardiovascular consequences of sleep apnea reported that changes in weight were related to an increase or decrease in sleep disordered breathing (SDB), and the relationship was stronger in males than in females [35]. Another interesting piece of information almost overlooked comes from studies on OSAS and male gonadal function. Many authors demonstrated that males with OSAS have reduced LH and testosterone with consequent hypogonadism [36-39]. Low testosterone levels and hypogonadism are in turn associated with diabetes and obesity [40-43]. Apparently as total body fat mass increases in the presence of low testosterone levels, hormone resistance develops for leptin and insulin. In agreement with these observations we described an increased leptin level in a population of diabetics suggesting the presence of leptin resistance [44]. More indirect data on the role of OSAS come from studies using the CPAP. In one of these studies on 30 OSAS subjects followed up to two months the use of CPAP modulated leptin and the other hormones involved in appetite regulation, independent of obesity [45].

The available data also demonstrate the existence of a series of anatomo-functional alterations acquired in Obesity and Diabetes than can further worsen OSAS thus creating a harmful vicious cycle: obesity reduces the pharyngeal lumen $[46,47]$ causes ovalization of the pharingeal shape [48] and the large abdomen causes a mass effect on tracheal traction [49]. Furthermore obesity increases daytime somnolence and inactivity [50-52] and as reported previously alters the hormonal balance interfering with appetite [53,54]. Of interest is the association of OSAS with the cardiovascular autonomic neuropathy of diabetes $[55,56]$, a condition that carries a high mortality rate [57]. The patency of the pharyngeal airways is dependent on the activity of central and peripheral neurones on the genioglossus and tensor palatini muscles, and it is legitimate to think that autonomic neuropathy can interfere with their function.

Unfortunately even the most sophisticated equipment cannot explore simultaneously all the intricacies of the glucose metabolism, the sleep rhythms and the respiratory and metabolic functions, thus the evidence is at most extremely suggestive but not conclusive. Furthermore due to the technical difficulties with the Continuous Positive Airway Pressure (CPAP) during sleep the compliance with this treatment is extremely poor and any the possibility to evaluate the results is compromised. It is also worth mentioning that any attempt to improve the quality and quantity of sleep with the benzodiazepine drugs inevitably results in a worsening of the respiratory pattern, thus probably reversing any beneficial effect

\section{CONCLUSION}

The knowledge of the interrelations linking respiratory 
disturbances, sleep and metabolic disorders is still in its infancy although the phenomenon has been acknowledged for many years. We cannot easily differentiate the effects of disturbed sleep of any cause from the oxygen deficit of OSAS, we do not know if there is a cause: effect response, if there is a threshold, to what extent the glucoregolatory and appetite regulatory systems can be affected, if there is a counter regulatory response to these disturbances, the short and long term effects. Most important we do not know for sure if the available treatment (CPAP) will cure the abnormality. On the other side, the disturbance of glucose metabolism connected with SDB/OSAS is not well categorized, and what we know is based mostly on the evaluation of fasting blood glucose, on the HbA1c level or the oral glucose tolerance test. Other relevant aspects of glucose metabolism, like glucose variability or the remote effects of nightly OSAS on postprandial blood glucose, have not been explored. Last, there was no consideration of the effect of minor degrees of respiratory obstruction on the various aspects of glucose metabolism. Any future study aiming to shed light over this puzzle should explore the sleep pattern, the breathing pattern and the blood glucose profile at the same time and for a prolonged time. We now have more powerful tools to study this problem, like the Holter monitors, and some metabolic monitors that in the near future will give more consistent answers to our doubts.

\section{REFERENCES}

[1] Craig, T.J., et al. (2005) How do topical nasal corticosteroids improve sleep and daytime somnolence in allergic rhinitis? The Journal of Allergy and Clinical Immunology, 116, 1264-1266. doi:10.1016/j.jaci.2005.10.009

[2] Kiely, J.L., et al. (2004) Intranasal corticosteroid therapy for obstructive sleep apnea in patients with coexisting rinitis. Thorax, 59, 50-55.

[3] Kerr, P., et al. (1992) The importance of nasal resistance in Obstructive Sleep Apnea Syndrome. Journal of Otolaryngology, 21, 189-195.

[4] McLean, H.A., et al. (2005) Effect of treating nasal obstruction on the severity of obstructive sleep apnoea. European Respiratory Journal, 25, 521-527. doi:10.1183/09031936.05.00045004

[5] DeFronzo, R.A. (2009) From the triumvirate to the ominous octet: A new paradigm for the treatment of type 2 diabetes mellitus. Diabetes, 58, 773-795. doi:10.2337/db09-9028

[6] Knitson, K.L., et al. (2007) The metabolic consequences of sleep deprivation. Sleep Medicine Reviews, 11, 163-178.

[7] Spiegel, K., et al. (2004) Sleep curtailment in healthy young men is associated with decreased leptin levels, elevated ghrelin levels, and increased hunger and appetite. Annals of International Medicine, 141, 846-850.

[8] Siegel, A., et al. (1999) Impact of sleep debt on metabolic and endocrine function. Lancet, 354, 1435-1439.

\section{doi:10.1016/S0140-6736(99)01376-8}

[9] Widén, E.I.M., et al. (1998) The relationship between first-phase insulin secretion and glucose metabolism. Acta Endocrinology, 127, 289-293.

[10] Cappuccio, F.P., et al. (2010) Quantity and quality of sleep and incidence of type 2 diabetes. Diabetes Care, 33, 414-420. doi:10.2337/dc09-1124

[11] NaJib, A.T., et al. (2003) A prospective study of self reported sleep duration and incident diabetes in women. Diabetes Care, 26, 380-384. doi:10.2337/diacare.26.2.380

[12] Tasali, E., et al. (2008) Slow-wave sleep and the risk of type 2 diabetes in humans. Proceedings of the National Academy of Sciences of the USA, 105, 1044-1049.

[13] Vgonitzas, A.N., et al. (2009) Insomnia with objective short seep duration is associated with type 2 diabetes. Diabetes Care, 32, 1980-1985. doi:10.2337/dc09-0284

[14] Bitz, C., et al. (2005) A paradoxical diurnal movement pattern in obese subjects with type 2 diabetes. Diabetes Care, 28, 2040-2042. doi:10.2337/diacare.28.8.2040

[15] Tiihonene, M., et al. (1993) The severity of obstructive sleep apnoea is associated with insulin resistance. Journal of Sleep Research, 2, 56-61. doi:10.1111/j.1365-2869.1993.tb00062.x

[16] Bergman, R.N. (1989) Toward physiological understanding of glucose tolerance: Minimal model approach. Diabetes, 38, 1512-1527. doi:10.2337/diabetes.38.12.1512

[17] Punjabi, N.M., et al. (2009) Alterations in glucose disposal in sleep-disordered breathing. American Journal of Respiratory and Critical Care Medicine, 179, 235-240.

[18] Punjabi, N.M., et al. (2002) Sleep disordered breathing and insulin resi stance in middle-aged and overweight men. American Journal of Respiratory and Critical Care Medicine, 165, 562-563.

[19] Mallon, L., et al. (2005) High incidence of diabetes in men with sleep complaints or short sleep duration. Diabetes Care, 28, 2762-2767. doi:10.2337/diacare.28.11.2762

[20] Seicean, S., et al. (2008) Sleep disordered breathing and impaired glucose metabolism in normal weight and overweight/obese individuals. Diabetes Care, 31, 10011006. doi:10.2337/dc07-2003

[21] Aronsohn, R.S., et al. (2010) Impact of untreated Obstructive Sleep Apnoea on Glucose control in Type 2 Diabetes. American Journal of Respiratory and Critical Care Medicine, 181, 507-513. doi:10.1164/rccm.200909-14230C

[22] Muraki, I., et al. (2010) Nocturnal intermittent hypoxia and the development of type 2 diabetes: The circulatory risks in communities study. Diabetologia, 53, 481-488. doi:10.1007/s00125-009-1616-0

[23] The Action to Control Cardiovascular Risk in Diabetes Study Group. (2008) Effect of intensive glucose lowering in type 2 diabetes. The New England Journal of Medicine, 358, 2545-2549. doi:10.1056/NEJMoa0802743

[24] McNicholas, A. and Bonsignore, M.R. (2010) Sleep apnea. European Respiratory Society Monograph, 50, 360-380.

[25] Kent, B.D., et al. (2011) Obstructive sleep apnea and inflammation: Relationship to cardiovascular co-morbidity. 
Respiratory Physiology and Neurobiology, 178, 475-481. doi:10.1016/j.resp.2011.03.015

[26] Taylor, R.S., et al. (2009) Systemic inflammation: A key factor in the pathogenesis of cardiovascular complications in obstructive sleep apnea syndrome? Thorax, 64, 631-636.

[27] Ip, M.S., et al. (2000) Clinical investigations serum leptin and vascular risk factors in obstructive sleep apnea. Chest, 118, 580-586.

[28] McArdle, M., et al. (2007) Metabolic risk factors for vascular disease in obstructive sleep apnea: A matched controlled study. American Journal of Respiratory and Critical Care Medicine, 175, 190-195.

[29] Wang. C.J., et al. (2010) Cytokines in the progression of pancreatic B-cell dysfunction. International Journal of Endocrinology, 2010, Article ID 515136. doi:10.1155/2010/515136

[30] Kolb, H. (2010) The global diabetes epidemic is a consequence of lifestyle induced low grade inflammation. Diabetologia, 53, 10-20. doi:10.1007/s00125-009-1573-7

[31] Smith, R., et al. (2002) What are obstructive sleep apnea patients being treated for prior to this diagnosis? Chest, 121, 164-172. doi:10.1378/chest.121.1.164

[32] Phillips, B.G., et al. (1999) Recent weight gain in patients with newly diagnosed obstructive sleep apnea. Journal of hypertension, 17, 1297-1300. doi:10.1097/00004872-199917090-00009

[33] Tatti, P. and Barber, A. (2011) Human Obesity: An overview of the mechanisms and the basis for treatment. Advances in Medicine and Biology, 15, 341-352.

[34] Spiegel, K., et al. (2004) Sleep curtailment in healthy young men is associated with decreased leptin levels, elevated ghrelin levels, and increased hunger and appetite. Annals of International Medicine, 141, 846-850.

[35] Newman, A.B., et al. (2005) Progression and regression of sleep-disordered breathing with changes in weight: The sleep hearth health study. Archives of International Medicine, 165, 2408-2413. doi:10.1001/archinte.165.20.2408

[36] Luboshitzky, B., et al. (2002) Decreased pituitary-gonadal secretion in men with obstructive sleep apnea. The Journal of Clinical Endocrinology and Metabolism, 87, 3394.

[37] Grunstein, R.R., et al. (1989) Neuroendocrine dysfunction in sleep apnea: Reversal by continuous positive airways pressure therapy. The Journal of Clinical Endocrinology and Metabolism, 68, 352-358. doi:10.1210/jcem-68-2-352

[38] Santamaria, J.D., et al. (1988) Reversible reproductive dysfunction in men with obstructive sleep apnoea. Clinical Endocrinology, 28, 461-470. doi:10.1111/j.1365-2265.1988.tb03680.x

[39] Luboshitzky, R., et al. (2001) Disruption of the nocturnal testosterone rhythm by sleep fragmentation in normal men. The Journal of Clinical Endocrinology and Metabolism, 86, 1134-1139. doi:10.1210/jc.86.3.1134

[40] Ando, S., Rubens, R., et al. (1984) Androgen plasma levels in male diabetics. Journal of Endocrinological Investigation, 7, 21-24.

[41] Haffner, S.M., et al. (1994) Insulin resistance, body fat distribution and sex hormones in men. Diabetes, 43 , 212-219. doi:10.2337/diabetes.43.2.212

[42] Vermeulen, A., et al. (1999) Testosterone, body composition and aging. Journal of Clinical Investigation, 22, 110-116.

[43] Pasquali, R., et al. (1991) Effect of obesity and body fat distribution on sex hormones and insulin in men. Metabolism, 40, 101-104. doi:10.1016/0026-0495(91)90199-7

[44] Tatti, P., et al. (2001) Leptin levels in diabetic and nondiabetic subjects. Endocrine, 15, 305-308.

[45] Harsh, I.A., et al. (2003) Leptin and grelin levels in patients with sleep apnea: Effect of CPAP treatment. European Respiratory Journal, 22, 251-257. doi:10.1183/09031936.03.00010103

[46] Brander, P.E., et al. (1999) Effect of obesity and erect/ supine posture on lateral cephalometry: Relationship to sleep-disordered breathing. European Respiratory Journal, 13, 398-402. doi:10.1183/09031936.99.13239899

[47] Hoffstein, V., et al. (1984) Lung volume dependence of pharyngeal cross-sectional area in patients with obstructive sleep apnea. American Review Respiratory Disease, 130, 175-178.

[48] Horner, R.L., et al. (1989) Pharyngeal size and shape during wakefulness and sleep in patients with obstructive sleep apnoea. Quarterly Journal of Medicine, 72, 719-735.

[49] Brown, L.K. (2002) A waist is a terrible thing to mind: Central obesity, the metabolic syndrome, and sleep apnea hypopnea syndrome. Chest, 122, 774-778. doi:10.1378/chest.122.3.774

[50] Fong, S.Y., et al. (2005) Comparing MSLT and ESS in the measurement of excessive daytime sleepiness in obstructive sleep apnoea syndrome. Journal of Psychosomatic Respiratory, 58, 55-60. doi:10.1016/j.jpsychores.2004.05.004

[51] Guilleminault, C., et al. (1988) Determinants of daytime sleepiness in obstructive sleep apnea. Chest, 94, 32-37. doi:10.1378/chest.94.1.32

[52] Seneviratne, U. and Puvanendran, K. (2004) Excessive daytime sleepiness in obstructive sleep apnea: Prevalence, severity, and predictors. Sleep Medicine, 5, 339-343. doi:10.1016/j.sleep.2004.01.021

[53] Phillips, B.G., et al. (2000) Increases in leptin levels, sympathetic drive, and weight gain in obstructive sleep apnea. American Journal of Physiology Heart Circuit Physiology, 279, H234-H237.

[54] Ozturk, L., et al. (2003) The association of the severity of obstructive sleep apnea with plasma leptin levels. Archives of Otolaryngology Head and Neck Surgery, 129, 538-540. doi:10.1001/archotol.129.5.538

[55] Keller, T., et al. (2007) Obstructive sleep apnea syndrome: The effect of diabetes and autonomic neuropathy. Journal of Physiology and Pharmacology, 58, 313-318.

[56] Ficker, J.H., et al. (1998) Obstructive sleep apnoea and diabetes mellitus: The role of cardiovascular autonomic neuropathy. European Respiratory Journal, 11, 14-19. doi:10.1183/09031936.98.11010014

[57] Clarke, B.F., et al. (1979) Diabetic autonomic neuropathy. Diabetologia, 17, 195-212. doi:10.1007/BF01235856 